\title{
Microlensing planets in M 22: Free-floating or bound?
}

\author{
R. de la Fuente Marcos and C. de la Fuente Marcos
}

Universidad Complutense de Madrid, Ciudad Universitaria, 28040 Madrid, Spain

Received 9 July 2001 / Accepted 31 August 2001

\begin{abstract}
We use detailed numerical simulations and theoretical estimates to show that, if confirmed, the unusually brief microlensing events observed by Sahu et al. (2001) in the field of the globular cluster M 22 might be explained as a result of microlensing by a population of clustered MACHOs, a dark cluster or RAMBO, not associated with the globular cluster. If real, this dark cluster would be located between M 22 and the Galactic bulge and could include at least $10^{6}$ substellar members with a typical size of 1-3 pc. Bound planets in wide or/and eccentric orbits are also able to reproduce the observed microlensing behaviour, but only if multiplanet systems (including large Kuiper-belt-like objects) are abundant, although, our calculations argue against the latter scenario as the ionization rate in M 22 is very high. Dynamically ejected or lone planets are, in principle, incompatible with the observational findings as they either escape their parent cluster in a relatively short time-scale after ejection or segregate toward the outskirts of the cluster. We discuss additional implications of the dark cluster scenario, including the existence of a population of RAMBOs toward the Galactic bulge.
\end{abstract}

Key words. celestial mechanics - stellar dynamics - dark matter - Galaxy: globular clusters: individual: NGC 6656 - globular clusters: general - gravitational lensing - planetary systems

\section{Introduction}

Gravitational microlensing occurs when the gravitational field of a massive object (the lens), located close to the observer's line-of-sight, bends the light from a distant object (the source) to generate two or more unresolved images. For a point mass event, the two images are not identical, they have different areas and opposite parities. Although the gravitational microlensing effect conserves surface brightness it alters the flux of the unresolved images as the relative positions of the lens, the source, and the observer change. These variations in flux translate into a measurable increase of the photometric magnitude (magnification). The effect does not depend on the photometric color used in the observations. Microlensing was proposed in 1986 by Paczyński as a method to detect compact baryonic dark matter in the halo of our Galaxy, but before the first events were discovered, Mao and Paczyński (1991) had already noted that is might be possible to detect planetary companions of the primary microlenses.

Stars or sub-stellar objects in globular clusters can act either as sources to detect MACHOS (Massive Astrophysical Compact Halo Objects) located along the line-of-sight or as lenses for more distant background stars (Jetzer et al. 1998). Under normal conditions, the probability of detecting microlensing events is very small but observing a globular cluster projected against the star-rich Galactic bulge increases this probability by a significant

Send offprint requests to: R. de la Fuente Marcos, e-mail: rfuente@ucmail.ucm.es amount. Monitoring the bulge stars for variability may then help to detect dim or dark objects within the star cluster. Using this technique, Sahu et al. (2001) (hereafter SM22) have recently presented evidence for the existence of free-floating planetary-mass objects in M 22 . They have detected six microlensing events that are completely unresolved in time. If these events indeed represent gravitational microlensing, the upper limit to the mass of the lens is less than $80 M_{\oplus}$ (Saturn mass is about 95 ). They interpret that these objects must be either free-floating, or at least several AUs from any stellar-mass objects, although they favour the free-floating hypothesis. If real, the total contribution of these free-floating planets to the mass of M 22 is estimated by these authors to be of the order of $10 \%$.

M 22 (NGC 6656) is an unusual star cluster with a diameter of about $18 \mathrm{pc}$ and ranking fourth in brightness among globulars, this heavily reddened metal-poor globular cluster is 12 Gyr old (Davidge \& Harris 1996). Its binary fraction is $0.01-0.03$ depending on the eccentricity as opposed to 0.12 , the binary fraction for nearby, solartype stars having similar mass ratios and periods (Cote et al. 1996). M 22 has a binary ionization rate as high as $\omega$ Centauri and very likely all its soft (long period) binaries have been disrupted by stellar encounters.

In this paper, we investigate how bound planets can induce single lens gravitational microlensing in star clusters. In Sect. 2, we present relevant results from numerical simulations. Different scenarios able to explain the SM22 
findings are introduced in Sect. 3 as well as relevant microlensing theory. Section 4 is a discussion.

\section{Models and results}

Here, we present partial results from a systematic longterm project aimed to study planetary dynamics in star clusters. Full details and results of our calculations will be presented elsewhere. Partial results from this program can be found in de la Fuente Marcos and de la Fuente Marcos $(1997,1998,2000,2001 a, b)$. In this paper, we will only provide the data required to support our interpretation of the results from SM22. Calculations have been carried out using a version of Sverre Aarseth's NBODY5 code (Aarseth 1994). This code includes the effect of the Galactic tidal field and the mass loss due to stellar evolution (Eggleton et al. 1989). Most of the models consider a realistic mass spectrum (Scalo 1986) in the range [0.08, 15.0] $M_{\odot}$. Spherical symmetry and constant star density or Plummer models are assumed for generating initial positions, with the ratio of the total kinetic and potential energy fixed to 0.25 ( 0.5 for virial equilibrium). Our choice of the initial ratio of kinetic to potential energy produces an initial contraction of the cluster and simulates violent relaxation. Several models ( $N=1000$ particles) include both a significant primordial binary fraction and realistic orbital elements for this binary population. The $N$ in our models ranges from 100 to 10000 . Although globular clusters are significantly more populated, our present results are consistent with very preliminary partial results from our models with $N \approx 50000$ and also with results from other authors (Laughlin \& Adams 1998, 2000; Adams \& Laughlin 2001; Smith \& Bonnell 2001; Bonnell et al. 2001), therefore we will apply them to globular clusters through this paper. Instead of computing the evolution of several cluster models with different populations and sizes we consider samples of models (10 or more for each $N$ ) only differing in the value of the seed number for generating initial conditions. For example, in one of our samples we choose an open cluster with $N=1000$ objects ( $40 \%$ binaries, $60 \%$ planetary systems) and a half-mass radius of about $0.9 \mathrm{pc}$. We select $40 \%$ primordial binaries as a plausible binary percentage for typical galactic clusters, with semi-major axis in the range 51.6-309.4 AU. For simplicity, the planetary systems studied in this research consist of one giant planet and its host star. Our giant planet populations have semi-major axes in the range $[0.5,60] \mathrm{AU}$, masses uniformly distributed in the range $0.1-8 M_{\mathrm{J}}\left(9.6 \times 10^{-4} M_{\odot}\right)$, and an initial eccentricity of 0.010 . Pericentre, nodes, and inclinations for both, planetary systems and binaries, as well as the eccentricities of the binaries, are chosen from a random (thermalized) distribution.

Our calculations show that a significant fraction of the planetary systems suffer modification of their primordial orbital elements as a result of complex gravitational interactions with binaries, single stars, and/or other planetary systems. Eccentricity modification is, by far, the most common event. Modification of the semi-major axis is not so frequent and just in a few cases an interaction results in a decrease of the orbital size. Most of the times, the semimajor axis changes as a result not only of external perturbations but also because of mass loss from the parent star. The more massive the planet, the higher the probability of being involved in an orbital modification event.

\section{Free-floating or bound?}

Before presenting different scenarios able to induce planetary microlensing we introduce some relevant equations from the gravitational microlensing theory. Following Gaudi \& Sackett (2000), let us consider a single lens, in this case the time-variable flux observed from a microlensed star is $F(t)=F_{0}\left(A(t)+f_{\mathrm{B}}\right)$, where $F_{0}$ is the unlensed flux of the star, $f_{\mathrm{B}}$ is the ratio of any unresolved, unlensed background light to $F_{0}$ (the "blend fraction"), and $A(t)$ is the magnification. The magnification for a single lens is given by $A_{0}=\left(2+u^{2}\right) /\left(u \sqrt{4+u^{2}}\right)$, where $u$ is the instantaneous angular separation of the source and the lens in units of the angular Einstein ring radius $\theta_{\mathrm{E}}$ of the lens that can be written as

$\theta_{\mathrm{E}}=\sqrt{\frac{4 G M}{c^{2}} \frac{D_{\mathrm{LS}}}{D_{\mathrm{OL}} D_{\mathrm{OS}}}}$

where $G$ is the gravitational constant, $M$ is the mass of the lens, $c$ is the speed of light, and $D_{\mathrm{LS}}, D_{\mathrm{OS}}, D_{\mathrm{OL}}$ are the lens-source, observer-source, and observer-lens distances, respectively. For a lens in M 22 and a source in the Galactic bulge, $D_{\mathrm{OL}}=2.6 \mathrm{kpc}$ and $D_{\mathrm{OS}}=8.2 \mathrm{kpc}$, therefore

$\theta_{\mathrm{E}} \approx 653 \mu \mathrm{as} \sqrt{\frac{M}{0.2 M_{\odot}}}$

In principle, if $u>1.5$, the magnification is not large enough to be measurable, although it depends strongly on the photometric errors. The characteristic timescale of one of these events is given by the Einstein time, $t_{\mathrm{E}}=\theta_{\mathrm{E}} D_{\mathrm{OL}} / v_{\mathrm{T}}$, where $v_{\mathrm{T}}$ is the transverse velocity of the lens relative to the observer-source line-of-sight. This timescale increases as $\sqrt{M}$, therefore the smaller the lens the shorter the duration of the microlensing event. If the lens is not a single object but it has a companion, the formalism for binary lenses should be used. The flux is still expressed by the same equation, but the magnification can no longer be calculated analytically and numerical techniques are required (Witt 1990). The minimum separation between a planet and its host star (in units of $\theta_{\mathrm{E}}$ ) for which planets can be discovered using microlensing is about 0.8 . For planetary separations roughly between 0.8 and 1.5 , the microlensing signature corresponds to a binary lens. For larger values of the separation, the planet generally acts as an independent lens. The angular separation given by Eq. (2) is equivalent to the spatial distance

$d_{\mathrm{E}} \approx 1.70 \mathrm{AU} \sqrt{\frac{M}{0.2 M_{\odot}}}$ 


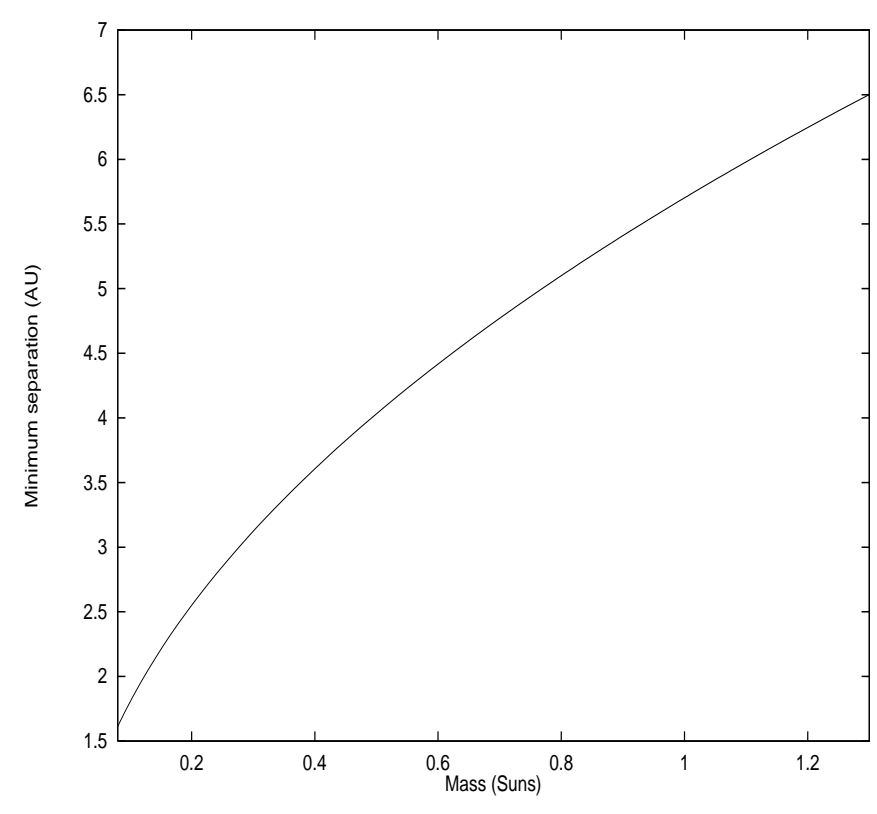

Fig. 1. Einstein ring radius of the lens multiplied by 1.5 as a function of the stellar mass for M 22. A bound planet with separation from its host star below the curve would induce double lens behaviour with contributions of both objects, planet and host star, clearly visible on the light curve. Above the curve, single lens behaviour is expected.

In order to obtain isolated short-duration events corresponding to planetary-class single-lens microlensing, the instantaneous separation between the planet and its host star must be larger than $1.5 d_{\mathrm{E}}$. This magnitude is plotted in Fig. 1, therefore virtually any planetary system (in M 22) with a separation between planet and host star wider than about $7 \mathrm{AU}$ (upper limit for massive primary) will induce a single lens microlensing event in exactly the same way a lone planet does. As an example and from the figure, if the primary has a mass of $0.3 M_{\odot}$, the minimum distance for single lens is $3.1 \mathrm{AU}$ at the $95 \%$ confidence level. For this hypothetical system any planet farther from the central star may induce single lens microlensing. More rigorous arguments using the full binary lens formalism give essentially the same results. Let us consider several configurations able to produce planetary-class single lens microlensing.

\subsection{Free-floating planets: Loners or escapees?}

\subsubsection{Lone planets}

SM22 interpret that their unresolved microlensing events have been probably induced by a free-floating planetarymass population located at the cluster core. However, existence of standalone planets poses some theoretical challenges. Star clusters tend to energy equipartition among stars of different mass. As a result of this process, the heavier stars will tend to slow down and sink toward the center of the cluster. Globular clusters are very old, therefore mass segregation has already been well established through the entire cluster. As an example from our calculations, for a $N=10000$ model and after about $500 \mathrm{Myr}$ mass segregation gives an average mass of about $1 M_{\odot}$ for the cluster core and about $0.5 M_{\odot}$ for the halo, and these values remain rather constant during the evolution of the model. Therefore, if they form, low-mass substellar objects should be a minority in the cluster core. On the other hand, standalone planets cannot be formed by accretion, therefore protostellar collapse and fragmentation is currently the only other alternative. Boss (2001) has shown that if the magnetic field tension effects are important, collapse and fragmentation of molecular clouds might be able to produce self-gravitating objects with Jovian (not Saturn or smaller) masses although this author terms the resulting objects as sub-brown dwarfs not planets. However, it seems that, regardless of whether the planets were formed via collapse or were formed via accretion and subsequently ionized, they would still not survive in the core of the cluster. Free-floating planets have recently been identified in Orion (Lucas \& Roche 2000; Zapatero Osorio et al. 2000) but their origin remains controversial as they can be escapees or even actual brown dwarfs.

\subsubsection{Wandering planets}

Dynamic or supernova driven ejection of planets is possible in star clusters (de la Fuente Marcos \& de la Fuente Marcos 1998) but the fraction of runaway planets is less than a few percent for the entire cluster, much smaller than the figure suggested by SM22. However, if the analysis is restricted to the core of a globular cluster which is a very high star density region this number rises to about $50 \%$, although ejected planets have very high characteristic velocities with the smaller planets escaping faster. The actual percentage depends on the semi-major axis: if it is $<3 \mathrm{AU}$ the ejection rate is $1 \%$ but it is almost $100 \%$ if the semiaxis is $>15 \mathrm{AU}$. On the other hand, in our models more than $70 \%$ of planetary escapees have a velocity $<25 \mathrm{~km} \mathrm{~s}^{-1}$ with an upper limit for the remaining fraction of about $200 \mathrm{~km} \mathrm{~s}^{-1}$. Fast or super-fast planetary escapees are always the result of strong multibody interactions inside the cluster core. Although for the core of M 22 the escape velocity is in the range $26-31 \mathrm{~km} \mathrm{~s}^{-1}$, ejections from the core are the result of strong close encounters with characteristic velocities much higher than the escape velocity. Therefore, it is very unlikely to be able to detect several of these events just by chance unless they are ejected planets moving almost parallel to the line-of-sight. In that case, the number of detected events might be consistent with the expected fraction of runaways from the cluster core. In this scenario, the detected objects would be moving toward us and likely located between M 22 and the Earth as they were ejected long ago. One can argue that this is practically impossible if the ejection is isotropic, as the fraction of planets moving sufficiently close to our line-of-sight would be incredibly 
tiny; however, ejections are only isotropical if they are the result of close encounters (likely at the cluster core). If planetary objects escape from the cluster as a result of evaporation (gradual increase in kinetic energy due to distant encounters) then they escape through the Lagrangian points, $L_{1}$ and $L_{2}$. If we consider lone planets formed by collapse, this is a plausible scenario only if a Lagrangian point is along our line-of-sight. However and although the lack of precise three-dimensional information makes it difficult to estimate, it is not very likely that one of the Lagrangian points might be projected toward the cluster core as the cluster is not observed against the Galactic Center.

\subsection{Solar-system-like systems}

Low eccentricity, distant sub-Saturnian planets similar to the gas giants (Uranus and Neptune) in our own Solar System are also able to generate microlensing events like the ones described in SM22. However, the probability of getting them involved in microlensing events is only significant if these planets are abundant (several per host star). On the other hand, Edgeworth-Kuiper-belt-like structures including Earth-sized objects in large numbers may also be able to induce brief microlensing events. In fact and from a strictly intuitive point of view, the relatively high number of planetary-class detections (6) as compared to a single classical (stellar) event argues in favor of a multiplanet scenario. However, low eccentricity primordial planetary orbits are very unlikely in a highly ionizing environment like M 22, at least in the cluster core. As pointed out before, planetary systems wider than 10-15 AU are catastrophically disrupted and due to mass segregation the most massive stars, and likely less favorable for planetary-class microlensing (Fig. 1), are dominant in the core and therefore the optical depth for bound planetary microlensing at the cluster core is not very significant.

On the other hand, it is also possible that the detected objects are projected against the core but they are actually part of the halo of M 22 where the star density is low enough to allow for relatively primordial, unperturbed (likely multiplanet) systems. Our results suggest that the percentage of planetary systems disrupted in the halo of a typical star cluster is very negligible, and the fraction that experiences significant variation $(>10 \%)$ in the orbital elements is a few percent and mainly connected with stellar evolution not dynamical interactions. If that is the case, the number of multiplanet systems similar to our Solar System in M 22, and likely in other globulars, could be much higher than expected. It is possible to argue against this latter scenario claiming that the optical depth (or probability) to lensing for the halo of M 22 is negligible. However, this could not be the case if we consider planetary objects instead of stars. Let us assume that the membership of M 22 is $10^{6}$, simulations suggest that for rich star clusters the core includes about $10 \%$ of the cluster total population, therefore we have about $10^{5}$ core stars (or planets if we assume that they exist) able to contribute to microlensing. As pointed out before, the actual number of planets available for single lens microlensing could be just a small fraction of that number, as low as a few percent. However, we are observing through the cluster halo. If we assume that the volume of the cluster is about $10^{3}$ the core volume and the core population is $10 \%$ of the total population, then the cluster average star density is about $1 / 100$ the core density. On the other hand, if we consider that the HST observed a region of radius 1 pc (core radius) and that the radius of M 22 is about $18 \mathrm{pc}$, then the number of halo stars included in an imaginary cylinder of radius $1 \mathrm{pc}$ and length $36 \mathrm{pc}$ could be estimated by considering that the average mass ratio between core and cluster is 1.5 . This number is about $30 \%$ the core population or 30000 stars, most of them low-mass. If we assume an average of 4 giant planets per star we found about 120000 objects available for microlensing. It implies that the optical depth to planetary companions in the halo of M 22 relative to its core is at least a factor 100 higher. Therefore, our simplified calculation suggests that, as regards single lens planetary microlensing, the halo of M 22 is dominant.

The probability $P$ or optical depth for a microlensing event is given by $P=\pi \theta_{\mathrm{E}} \sigma$, where $\sigma$ is the surface density of objects. Therefore, the probability for a core-induced classical (stellar) microlensing event is $P_{\mathrm{c}} \propto M_{\mathrm{c}} \sigma_{\mathrm{c}}$, where $M_{\mathrm{c}}$ is the average mass for stars in the core, and $\sigma_{\mathrm{c}}$ is the surface density of stars toward the cluster core. In addition, the probability for a halo-induced planetary microlensing event will be $P_{\mathrm{p}} \propto M_{\mathrm{p}} \sigma_{\mathrm{p}}$, with $M_{\mathrm{p}}$ the average mass for planets in the halo and $\sigma_{\mathrm{p}}$ the surface density of planets toward the cluster halo; this can be calculated by using $\sigma_{\mathrm{p}}=N_{\mathrm{p}} \sigma_{\mathrm{h}}$, where $N_{\mathrm{p}}$ is the number of planets per halo star and $\sigma_{\mathrm{h}}$ is the surface density of stars toward the cluster halo. To estimate the actual number of planets per star we may use $\sigma_{\mathrm{c}} / \sigma_{\mathrm{h}} \sim 3$ and $M_{\mathrm{c}} / M_{\mathrm{p}} \sim 10^{3}$. On the other hand, if as suggested by the observations the proportion of available planets/stars for microlensing is 6 to 1 then the average number of planetary-size companions per halo star could be as high as 20000 if we consider sub-Saturnian objects or about 2000000 if the lenses are Earth-sized. In any case, the results of this quantitative analysis makes it difficult to attribute the observed events to planetary objects in M 22 unless planets in globular clusters (or the early Universe for that matter) formed in a fundamentally different manner than planets in the Galactic disk.

\subsection{Eccentric planets}

If the objects detected by SM22 are actually located in the core of M 22, low-eccentricity primordial planetary orbits are very unlikely if the initial semi-major axis is larger than about $0.5 \mathrm{AU}$, as they should be, otherwise a double lens microlensing event must be recorded, Fig. 1. Our numerical results show that within the cluster lifetime and 
for high star density environments $\left(10^{3}-10^{4} M_{\odot} / \mathrm{pc}^{-3}\right)$ like the core of M 22 about $50 \%$ of systems are disrupted with the remaining fraction being characterized by high eccentricities $(e>0.3)$. A large fraction of planets in very elongated orbits increases the probability of single lens microlensing but not very significantly due to random orientations.

\subsection{Dark clusters: MACHOs and RAMBOs}

Finally, the events reported by SM22 may have been induced by non-M 22-related substellar objects located along the line-of-sight. Although current Massive Astrophysical Compact Halo Objects (MACHOs) observational results toward the Large Magellanic Cloud (LMC) exclude brown dwarfs as the primary constituent of the halo (Alcock et al. 1998), any hypothetical population of free-floating halo (single or binary) brown dwarfs should have been born elsewhere, namely in massive dark clusters or Robust Associations of Massive Baryonic Objects (RAMBOs) (Moore \& Silk 1995). The dynamics of these objects must be quite different from that of typical star clusters. With a very narrow mass range, the evaporation of these RAMBOs should be very slow as predicted by the evolution of mono-component cluster models (de la Fuente Marcos 1995). These very long-lived objects may exist in large numbers. On the other hand, the microlensing rate is $\Gamma \propto \sqrt{M} N$, where $M$ is the mass of the lens and $N$ the number of available lensing objects. SM22 found a single stellar microlensing event (very likely M 22-related) and six unresolved events. If they are indeed due to substellar objects in the field they must be clustered and a naive estimate suggests that the dark cluster population must be at least 100 times larger than the core population of M 22, with the same apparent diameter. It means that the dark cluster might include $10^{7}-10^{8}$ members with a total mass of $10^{4}-10^{5} M_{\odot}$. In principle, the object could be located in front of or behind M 22. For the same duration (upper limit, 0.8 days), the lens is more massive if the dark cluster is near the Galactic bulge and if its transverse velocity relative to the observer-source line-of-sight is higher. In any case the objects are well below 13 Jovian masses. The existence of a clustered thick disk-like component of dark matter in the Milky Way has been suggested by Sanchez-Salcedo (1997, 1999) and Kerins (1997).

\section{Discussion}

Although one can argue that the most likely explanation of the brief events toward M 22 is simply that they are not due to microlensing but, for example, to stellar variability, we will not include this conclusion in our discussion. Rather than consider astrophysical explanations as stellar variability, we will focus our discussion, assuming that the events are due to microlensing, on the astrodynamical (or kinematical) explanations. Our analysis suggests that, if stellar variability is excluded, only two scenarios are able to explain the unresolved microlensing events observed toward M 22: a cluster halo very rich in multiplanet systems (but Solar System-like) or a chance alignement with a dark cluster or RAMBO. The upper limit for the mass of the substellar lenses found by SM22 is about 80 times that of Earth. In the first scenario, as microlensing detection is totally by chance it means that the distribution of masses of the planetary-mass population in M 22 peaks around that value with a very likely negligible fraction of planets above it. However, in the Galactic disk the fraction of substellar objects with Jovian (or higher) masses is not negligible. If the objects are indeed bound, most planetary systems in M 22 are dominated by planets of about Saturn's mass. Preferential formation of low-mass giant planets in globular clusters can be explained as a result of poor metallicity and shorter lifetime of protoplanetary disks. In any case, planetary formation in the early Universe seems to be rather different from current one with super-Jupiters being more numerous now. On the other hand, as SM22 detected only one classical (stellar) microlensing event but six unexpectedly brief events it means that M 22 is rather rich in planetary-mass objects. As pointed out before, a planet in a low-eccentricity, short semi-major axis orbit will induce a double lens behaviour with both objects (planet \& host star) contributing to the magnification. On the other hand, a planet in a wide but not eccentric orbit will be able to induce a photometric behaviour like the one observed by SM22 but these objects are unlikely in the core of a globular cluster. If the lenses are located at the cluster halo the relatively high rate of detections suggests that multiplanet systems are very common in globular clusters and that giant planets in wide orbits are dominant as in our own planetary system.

The dark cluster scenario is also able to explain the observations. The only problem posed by this explanation is the mass of the cluster members. From the Einstein time equation, the upper limit for the mass of the lenses is a few Jovian masses. From a strictly theoretical point of view it is difficult to explain how such an object can form. On the other hand, the mass of the dark cluster might span the range $10^{4}-10^{5} M_{\odot}$. This is consistent with primordial (pre-Galactic) origin of MACHO clusters as cosmological considerations of the minimum Jeans mass suggest a typical pre-Galactic mass scale of $10^{4}-10^{6} M_{\odot}$. If there exist many more of these dark clusters, the microlensing statistics would be essentially unchanged from the unclustered case (Metcalf \& Silk 1996), therefore it would be a really serendipitous discovery. This is consistent with the lack of an analogous population of events toward the LMC (Alcock et al. 1998).

In this paper we have presented several plausible alternatives to the free-floating planets interpretation of the microlensing events observed by SM22. Our analysis is not meant to provide a unique or complete model either to explain microlensing in M 22 or in any other star cluster. Instead we simply point out different but compatible scenarios able to generate the same photometric signatures as detected by SM22. Our analysis can be easily tested by 
surveying low ionization rate globular clusters like M 71, M 4, or NGC 3201 in which, if they form, a fraction of low eccentricity, short semi-major axis planetary systems is likely to survive and be able to contribute to (binary) microlensing. If the observation of other clusters is not successful in finding microlensing events then the dark cluster scenario is the only plausible scenario. In addition, a comparison between microlensing data from core and off-core observations of M 22 may also help to clarify this matter, and follow-up HST observations can shed new light on this tentative discovery.

Acknowledgements. We thank Dr. S. J. Aarseth for providing his computer code and Dr. K. C. Sahu for some remarks on his results. The authors thank the Department of Astrophysics of Universidad Complutense de Madrid (UCM) for allotting excellent computing facilities. We also thank the referee, Scott Gaudi, for his rapid and very helpful report. Part of the calculations were performed on the SGI Origin 2000 of the "Centro de Supercomputación Complutense" through the UCM project "Dinámica Estelar y Sistemas Planetarios" (CIP 454). In preparation of this paper, we made use of the ASTRO$\mathrm{PH}$ e-print server and the NASA Astrophysics Data System.

\section{References}

Aarseth, S. J. 1994, in Galactic Dynamics and $N$-body Simulations, ed. G. Contopoulos, N. K. Spyrou, \& L. Vlahos (Springer Verlag, Berlin), 277

Adams, F. C., \& Laughlin, G. 2001, Icarus, 150, 151

Alcock, C., Allsman, R. A., Alves, D., et al. 1998, ApJ, 499, L9

Bonnell, I. A., Smith, K. W., Davis, M. B., \& Horne, K. 2001, MNRAS, 322, 859

Boss, A. P. 2001, ApJ, 551, L167

Cote, P., Pryor, C., McClure, R. D., Fletcher, J. M., \& Hesser, J. E. 1996, AJ, 112, 574
Davidge, T. J., \& Harris, W. E. 1996, ApJ, 462, 255

Eggleton, P. P., Fitchett, M. J., \& Tout, C. A. 1989, ApJ, 347, 998

de la Fuente Marcos, R. 1995, A\&A, 301, 407

de la Fuente Marcos, C., \& de la Fuente Marcos, R. 1997, A\&A, 326, L21

de la Fuente Marcos, C., \& de la Fuente Marcos, R. 1998, NewA, 4, 21

de la Fuente Marcos, R., \& de la Fuente Marcos, C. 2000, in Stellar Clusters and Associations: Convection, Rotation, and Dynamos, ed. R. Pallavicini, G. Micela, \& S. Sciortino (San Francisco), ASP Conf. Ser., 198, 183

de la Fuente Marcos, C., \& de la Fuente Marcos, R. 2001a, A\&A, 371, 1097

de la Fuente Marcos, C., \& de la Fuente Marcos, R. 2001b, in Modes of Star Formation and the Origin of Field Populations, ed. E. Grebel, \& W. Brandner (San Francisco), ASP Conf. Ser., in press

Gaudi, D. S., \& Sackett, P. D. 2000, ApJ, 528, 56

Jetzer, P., Straessle, M., \& Wandeler, U. 1998, A\&A, 336, 411

Kerins, E. J. 1997, A\&A, 322, 709

Laughlin, G., \& Adams, F. C. 1998, ApJ, 508, L171

Laughlin, G., \& Adams, F. C. 2000, Icarus, 145, 614

Lucas, P. W., \& Roche, P. F. 2000, MNRAS, 314, 858

Mao, S., \& Paczyński, B. 1991, ApJ, 374, 37

Metcalf, R. B., \& Silk, J. 1996, ApJ 464, 218

Moore, B., \& Silk, J. 1995, ApJ, 442, L5

Paczyński, B. 1986, ApJ, 304, 1

Sahu, K. C., Casertano, S., Livio, M., et al. (SM22) 2001, Nature, 411, 1022

Sánchez-Salcedo, F. J. 1997, ApJ, 487, L61

Sánchez-Salcedo, F. J. 1999, MNRAS, 303, 755

Scalo, M. J. 1986, Fundam. Cosmic. Phys., 11, 1

Smith, K. W., \& Bonnell, I. A. 2001, MNRAS, 322, L1

Witt, H. J. 1990, A\&A, 236, 311

Zapatero Osorio, M. R., Béjar, V. J. S., Martín, E. L., et al. 2000, Science, 290, 103 